\title{
Terahertz bandwidth photonic Hilbert transformers based on synthesized planar Bragg grating fabrication
}

\author{
Chaotan Sima*, J.C.Gates, C.Holmes, P.L.Mennea, M.N.Zervas, and P.G.R.Smith \\ Optoelectronics Research Centre, University of Southampton, Southampton SO17 1BJ, United Kingdom \\ *Corresponding author: Chaotan.Sima@soton.ac.uk
}

\begin{abstract}
Terahertz bandwidth photonic Hilbert transformers are proposed and experimentally demonstrated. The integrated device is fabricated via a direct UV grating writing technique in a silica-on-silicon platform. The photonic Hilbert transformer operates at bandwidths of up to $2 \mathrm{THz}(\sim 16 \mathrm{~nm})$, which is at least ten fold greater bandwidth than any previously reported experimental approaches. Achieving this performance requires detailed knowledge of the system transfer function of the direct UV grating writing technique, this allows improved linearity, and yields THz bandwidth Bragg gratings with improved spectral quality. By incorporating a flat-top reflector and Hilbert grating with a waveguide coupler an ultra wideband alloptical single-sideband filter is demonstrated. (C) 2013 Optical Society of America
\end{abstract}

All-optical devices offer a wide range of applications in telecommunication and information processing as they can provide operational bandwidths and speeds far beyond current electronic technologies. In the conventional electronic domain, Hilbert transform (HT) techniques have been widely used in singlesideband (SSB) modulation applications for improving performance in terms of required power, enhancing spectral efficiency and reducing fading. Devices have been implemented in both analogue and digital systems [1]. In the all-optical field, photonic Hilbert transformers (PHTs) provide wide bandwidths and high speeds for applications including the characterization of broadband microwave signals [2] and all-optical SSB filtering [35]. In addition they also reduce dispersion related artifacts such as optical signal power fading [3]. PHTs have been practically realized via various methods, e.g. using fiber Bragg gratings (FBGs) [6, 7], and optical ring resonators [8]. It has been estimated that these experimental approaches are limited to operation bandwidths $<100 \mathrm{GHz}$ due to intrinsic complexity of approaches via discrete components or the restrictions of current FBG fabrication [4]. Terahertz bandwidth PHTs were numerically presented using long period gratings and core/cladding mode interaction $[9,10]$. However, the structural complexity and large thermal sensitivity of long-period gratings pose significant challenges to practically realizing such a device.

In this work, we experimentally demonstrate a direct and simple route to realizing Bragg grating based PHTs with operation bandwidths up to $2 \mathrm{THz}$ $(\sim 16 \mathrm{~nm})$, over an order of magnitude larger than any previously reported experimental approaches. We use phase-modulated direct UV grating writing in a silica-on-silicon planar format [11], which provides an approach realizing compact optical modules for integrated optical applications. Furthermore, the linearity of this fabrication process is improved by considering the photosensitivity response and improving the spectral quality. PHTs have also been integrated to implement ultra wideband all-optical
SSB filters, without the need for optical circulators. These devices have the benefit of processing Tbit/s serial optical communication signals, being compact, with fiber compatibility, thermal stability and could be used alongside etched silica-on-silicon devices.

The conventional Hilbert transform $\mathrm{H}[\mathrm{g}(\mathrm{t})]$ of a onedimensional real signal $\mathrm{g}(\mathrm{t})$ is defined as [1]:

$$
H[g(t)]=\hat{g}(t)=g(t) *{ }_{\pi t}^{1}={ }_{\pi}^{1} P \int_{-\infty}^{\infty} g(\tau) d \tau
$$

where $\mathrm{P}$ stands for the Cauchy principal value [1]. The symbol * denotes the convolution of $\mathrm{g}(\mathrm{t})$ and1/nt. The Fourier transform of the kernel of the Hilbert transform is

$$
H(\omega)=-j \operatorname{sgn}(\omega)
$$

where $\omega$ is the angular frequency and where $\operatorname{sgn}(\omega)$ is the sign function (which is +1 for $\omega>0$, 0 for $\omega=0$ and -1 for $\omega<0$ ).

In any practical implementation of Hilbert transform, the output signal is a delayed and somewhat distorted version of the ideal HT of the input signal. This is a consequence of the fact that the frequency response of a practical and physically realizable HT will be band-limited with a bandwidth $\Delta \mathrm{f}$ and a smooth transition in phase, as illustrated in Fig. 1. For a physically realizable PHT, the practical grating apodization profile has been given in [6]:

$$
\Delta n(z) \propto \begin{gathered}
\sin ^{2}\left[\pi n_{\text {eff }} \Delta f\left(z-z_{0}\right) c\right] \\
z-z_{0}
\end{gathered}
$$

Where $\mathrm{n}_{\text {eff }}$ is the grating effective refractive index, $\mathrm{c}$ is the light speed in vacuum, $\mathrm{z}$ is the grating length, $\mathrm{z}_{0}$ is the zero-crossing point in the apodization function, and $\Delta \mathrm{f}$ is the operative bandwidth. From Eq. (3), the full operation spectral bandwidth of the PHT is inversely proportion to the size of the main apodization lobe $\Delta z$ [6], as shown in Fig. 2(a). The operation bandwidth is related to $\Delta \mathrm{z}$ by: 


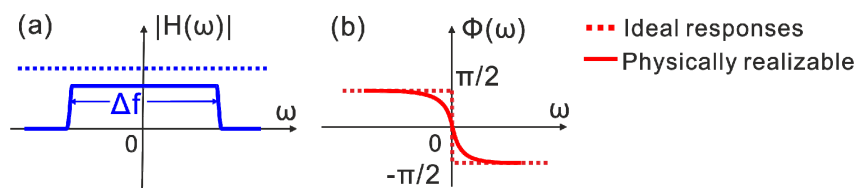

Fig. 1. Schematic of the (a) amplitude and (b) phase responses of a physically realizable HT (solid lines) compared to those of the ideal HT (dashed lines) in the frequency domain.
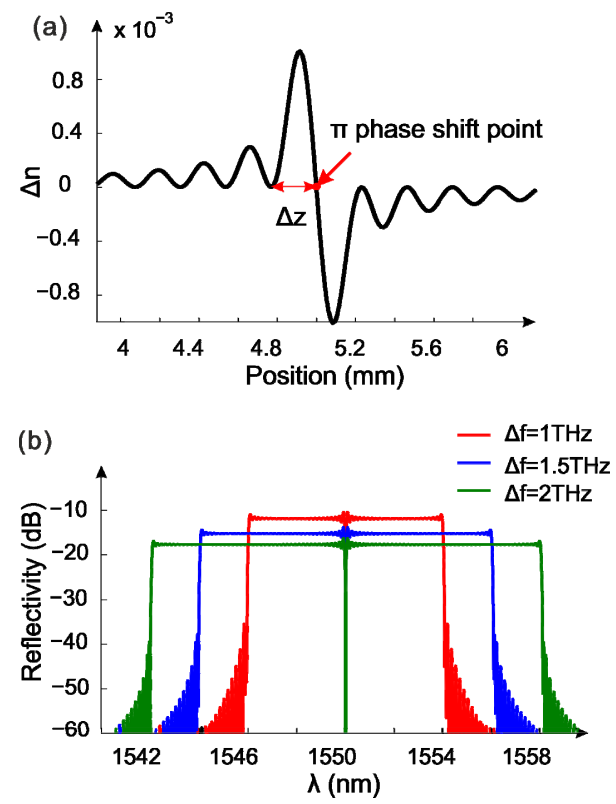

Fig. 2(a) The central apodization profile of the Bragg grating for a $1 \mathrm{THz}$ bandwidth PHT; (b) modeled amplitude responses of photonic Hilbert Transformers with increasing terahertz bandwidths.

$$
\Delta f=\frac{c}{n_{e f f} \Delta z}
$$

Therefore a broader processing bandwidth can be obtained using narrower apodization sidelobes. The device output for the proposed structure was modeled using the transfer matrix method. The following parameters were used: grating length $\mathrm{z}=10 \mathrm{~mm}, \mathrm{n}_{\text {eff }}$ $=1.45$ and the maximum refractive index modulation depth was $\Delta \mathrm{n}=9 \times 10^{-4}$. Figure 2 (a) presents the central section of the apodization profile for a $1 \mathrm{THz}$ bandwidth PHT scenario, showing the main sidelobe width $\Delta \mathrm{z}$ and the $\Pi$ phase shift position. Figure 2(b) illustrates the reflectivity spectra of the proposed Bragg gratings, with bandwidths of $1 \mathrm{THz}, 1.5 \mathrm{THz}$ and $2 \mathrm{THz}$ respectively. According to Eq. (4), the corresponding apodization profile main lobe sizes, $\Delta z$, were around $207 \mu \mathrm{m}, 138 \mu \mathrm{m}$ and $104 \mu \mathrm{m}$, respectively.

For the current FBG fabrication technologies using phase masks, the UV writing spot allows grating apodization profiles with spatial resolutions of $\sim 100 \mu \mathrm{m}$ to be written, hence the previously reported PHTs were limited in operation bandwidth to $<100 \mathrm{GHz}$ [10]. To overcome the restrictions and to obtain larger bandwidths, we use an alternative fabrication approach.

The proposed planar structures were fabricated using the direct UV grating writing (DGW) technique with phase modulation [11]. This method involves focusing two crossed laser beams $(\lambda=244 \mathrm{~nm})$ into the photosensitive core layer of a planar sample. Constant translation of the sample and modulation of the fringe pattern position defines the channel waveguide and simultaneously allows the creation of the grating structures. Phase modulation of one beam in the interferometer, achieved via an electrooptic modulator (EOM), and variation of the duty cycle is used to manipulate the fringe pattern and thus control the parameters of the Bragg gratings
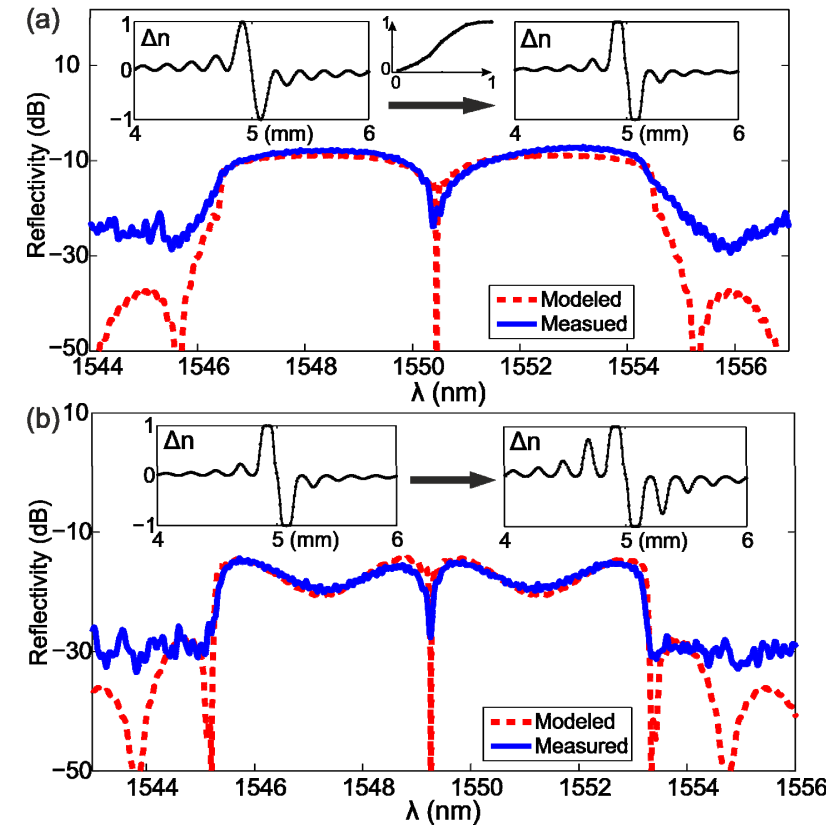

Fig. 3(a) Modeled data (red dash) and measured data (blue line) of the reflectivity of the initial directly designed PHT, the insets show that the practical $\Delta \mathrm{n}_{\mathrm{ac}}$ profile was different from the ideal $\Delta \mathrm{n}_{\text {ac }}$ profile, due to the nonlinear effect in the fabrication system; (b) shows the responses of the fabricated PHT, using the $\Delta \mathrm{n}_{\mathrm{ac}}$ profile in the inset, where the $\Delta \mathrm{n}_{\mathrm{ac}}$ value outside two main peaks was deliberately enlarged.
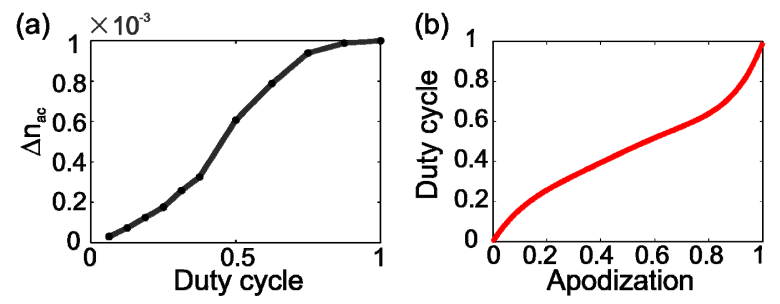

Fig. 4(a) Experimental data showing relationship between the maximum grating refractive index contrast $\Delta \mathrm{n}_{\mathrm{ac}}$ and duty cycle, tested with a series of uniform planar Bragg gratings; (b) the curve of the synthesis function for linearity enhancement in the DGW fabrication system. 
and waveguides. The resulting spot size is $\sim 6 \mu \mathrm{m}$ in diameter, providing the unique ability over traditional FBG writing techniques to manipulate the grating's structure at the sub 10 micron level.

Considering the maximum grating refractive index modulation depth $\Delta \mathrm{n}_{\mathrm{ac}}$ achieved in the DGW technique and given that the UV fluence is kept always constant, the magnitude of the local refractive index modulation (apodization profile) is defined by the phase modulation duty cycle. Conventionally it is assumed that the refractive index modulation depth $\Delta \mathrm{n}_{\mathrm{ac}}$ is linearly dependent on the duty cycle [11]. Using highly photosensitive glasses allows for rapid fabrication and thus fast translation speeds. This is desirable as it reduces the effects of drifts in the interferometer system. However, fast translation requires high frequency modulation, and that presents challenges to the control electronics of the system. In particular, slewrate limitations on the EOM driver can result in distortions of the actual grating compared to the target design. This gives a nonlinear relationship between the duty cycle and the $\Delta \mathrm{n}_{\mathrm{ac}}$ (as shown in figure 4a). The requirement for accurate fluence matching [12] also demands precise knowledge of the system response.

In this work, the impact of the $\Delta \mathrm{n}_{\mathrm{ac}}$ versus duty cycle linearity of the DGW fabrication technique was investigated and verified with grating responses. Figure 3(a) shows modeled data (red dots) and measured spectrum (blue line) of the initial directly designed grating for a $1 \mathrm{THz} \mathrm{PHT}$ and the insets in Fig. 3(a) illustrate the corresponding refractive index modulation profile. The current apodization profile is dissimilar to the targeted profile (shown in Fig. $2(\mathrm{a})$ ). This is a result of the saturation induced nonlinearity of the UV writing process, the top of the main lobe is truncated and the grating response is compromised. To further explore the effect of the nonlinear UV writing process, a windowing function was introduced which exclusively doubles the index contrast value outside the two main peaks in the $\Delta \mathrm{n}_{\mathrm{ac}}$ profile and significantly distorts the original profile. The modeled data and the measured results of the grating reflectivity are shown in Fig. 3(b) and the $\Delta \mathrm{n}_{\mathrm{ac}}$ profile resulting from the windowing function is presented in the right inset of Fig. 3(b).

The data in Fig. 3 demonstrates that the UV writing process's nonlinear response results in a significant departure from the target grating response. Figure 4(a) shows the transfer function of the entire fabrication system, demonstrating a nonlinear relationship between maximum grating refractive index contrast $\Delta \mathrm{n}_{\mathrm{ac}}$ and duty cycle. The data in Fig. 4(a) was obtained by characterizing the maximum grating refractive index contrast $\Delta \mathrm{n}_{\mathrm{ac}}$ of a series of $1 \mathrm{~mm}$ uniform Bragg gratings fabricated with increasing duty cycle. The inverse function of that in Fig. 4(a) was utilized in the DGW process to counterbalance this nonlinearity effect, as shown in Fig. 4(b). By using this synthesis function, substantial improvement in terms of the spectral
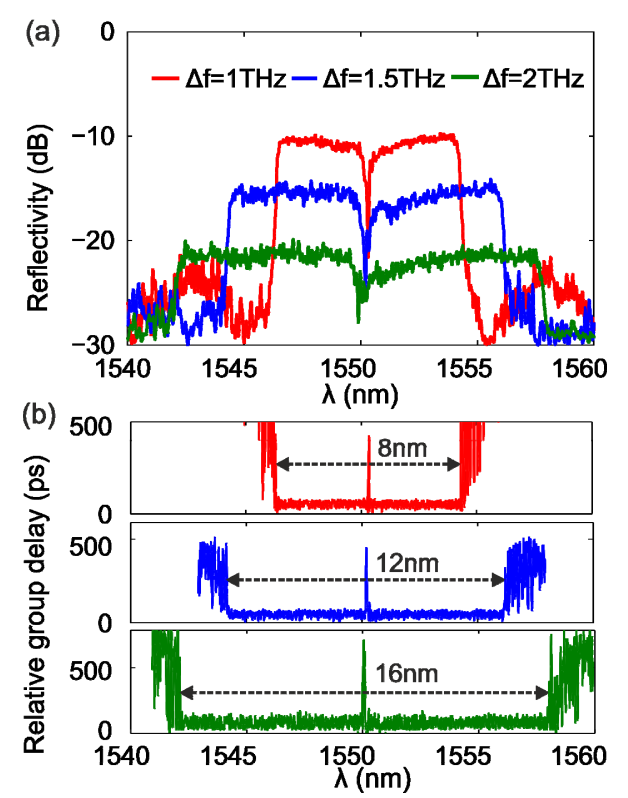

Fig. 5.(a) The measured reflectivity spectra of the fabricated Bragg gratings implementing $1 \mathrm{THz}, 1.5 \mathrm{THz}$ and $2 \mathrm{THz}$ bandwidth PHTs; (b) Relative group delay data of the planar Bragg gratings.

quality was achieved. The fabricated gratings spectral responses are shown in Fig. 5. This synthesized fabrication technique, correcting for the initial nonlinear response, is a significant step for making gratings with complex profiles.

The fabricated devices had three layers of silica deposited on a silicon substrate, and consisted of a thermal oxide layer (lower clad), core and an upper clad. The thickness of the core was $\sim 6 \mu \mathrm{m}$, which ensured vertical single mode operation. The Bragg grating sections were $10 \mathrm{~mm}$ long, and the $\Delta \mathrm{n}_{\mathrm{ac}}=$ $9 \times 10^{-4}$. Figure 5 shows the measured (a) reflectivity and (b) relative group delay data of the fabricated devices with terahertz bandwidths. The grating reflectivity was measured using an optical spectrum analyzer (OSA), $3 \mathrm{~dB}$ coupler and an erbium doped fiber amplified spontaneous emission (ASE) source. The measurement system has limited dynamic range resulting in a noise floor of about $-30 \mathrm{~dB}$ of the reflectivity measurement. The group delay data was measured via a modulation phase-shift method and shows the phase uniformity of the gratings $[5,13]$. In Fig. 5(a), the PHTs have flattop amplitude responses, with $<1 \mathrm{~dB}$ ripples for the $1 \mathrm{THz}$ bandwidth PHT. Fig. 5(b) indicates the $\sim \Pi$ phase shift at the central dip of the amplitude spectra. Active phase control, e.g. thermal tuning [3], could be used to compensate the PHT phase imbalance. The series of initial proofof-concept devices demonstrate the $8 \mathrm{~nm}(\sim 1 \mathrm{THz})$, $12 \mathrm{~nm}(\sim 1.5 \mathrm{THz})$ and $16 \mathrm{~nm}(\sim 2 \mathrm{THz})$ operation bandwidth PHTs respectively.

To investigate the fabricated devices performance, the silica-on-silicon integrated format was used to build an all-optical SSB filter [5], composed of an Xcoupler, flattop reflector, and a PHT. A sinc-apodized Bragg grating was utilized as the flattop reflector 


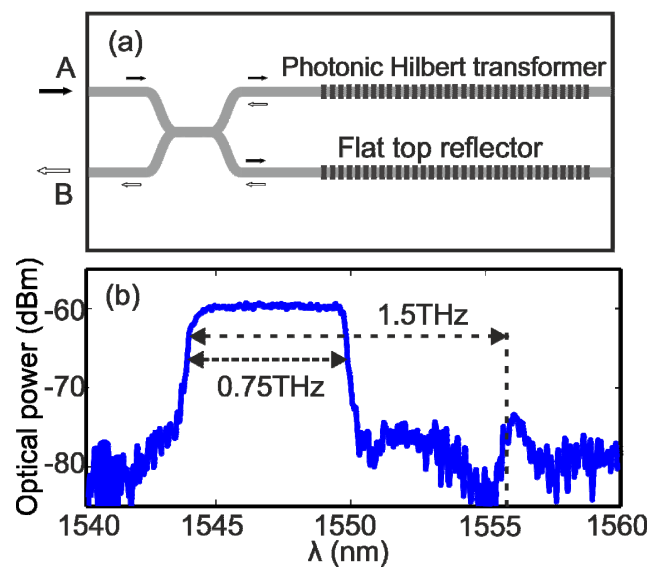

with the same length and similar bandwidth to the PHT. The reflected optical signal from the two gratings undergoes constructive and destructive interference, thereby suppressing one side band and enhancing the other. The relative optical phase difference of the two signals could be further finetuned by a small micro heater present on one arm of the device [5], although in this work that was not implemented. The X-coupler length was $\sim 7.5 \mathrm{~mm}$, with a crossing angle of 1.8 degrees. The crossing angle of the X-coupler was further optimized from the previous work [5] yielding an improved signal balance of the intensity of signals reflected from the two arms. The spacing between the X-coupler arms was $250 \mu \mathrm{m}$ to provide coupling with a standard two-port fiber $\mathrm{v}^{-}$ groove assembly. The interferometric structure is schematically shown in Fig. 6(a). Such a structure enables the incorporation of reflective Bragg gratings in more complex optical systems without the use of an optical circulator.

Figure 6(b) shows the output optical power spectra directly from port $\mathrm{B}$ of the device, incorporating the 1.5 THz bandwidth PHTs, with input at port A, measured using an OSA and a broadband ASE source. Optical signals among the spectral band below the central wavelength $(1544-1550 \mathrm{~nm})$ have a ripple-less pass band while signals within the other band $(1550-1556 \mathrm{~nm})$ are eliminated and the level of suppression is $\sim 20 \mathrm{~dB}$. Figure $6(\mathrm{~b})$ also confirms that the operation bandwidth of the all-optical SSB filter is $\sim 0.75 \mathrm{THz}$. The total insertion loss of the actual devices was $\sim 20 \mathrm{~dB}$ due to excess coupling loss, propagation loss in the $\mathrm{X}$-coupler and a rather low grating reflectivity. This could be potentially reduced by improving the $\mathrm{X}$-coupler structures and using stronger gratings designed by inverse scattering methods [14].

This technique also has potential in applications of optical Nyquist pulse transmission in optical communication networks $[15,16]$. The use of integrated devices with terahertz bandwidth and sharp edge spectral features fabricated by this method provides a route for all-optical Nyquist pulse generation with ultra-wideband features.

In conclusion, we have proposed and experimentally demonstrated a series of terahertz bandwidth photonic Hilbert transformers, using planar Bragg gratings. PHT devices with $1 \mathrm{THz}, 1.5$ $\mathrm{THz}$ and $2 \mathrm{THz}$ operation bandwidths were fabricated via a novel linearity-enhanced direct UV grating writing technique. This technique allows for precise engineering of the amplitude and phase response of gratings with complex structures at the sub-10 micron level. The PHTs were also integrated with directional couplers and flat top reflectors to perform ultra-wideband all-optical SSB filtering. This approach would allow compact devices to be implemented for processing ultra-wideband optical signals such as Nyquist pulses with terahertz bandwidths.

This work was supported by Engineering and Physical Sciences Research Council (EPSRC), UK and China Scholarship Council, China. 


\section{References}

1.S. L. Hahn, "The Transforms and Applications Handbook," 2nd ed., A. D. Poularikas, ed. (CRC Press LLC, Boca Raton, Fla., 2000).

2. H. Emami, N. Sarkhosh, L. A. Bui, and A. Mitchell, "Amplitude independent RF instantaneous frequency measurement system using photonic Hilbert transform," Opt. Express, 16, 13707-13712 (2008) .

3. Z. Li, H. Chi, X. Zhang, and J. P. Yao, "Optical singlesideband modulation using a fiber-Bragg-grating-based optical Hilbert transformer," IEEE Photon. Technol. Lett., 23, 558-560 (2011).

4. X. Wang, M. Hanawa, K. Nakamura, K. Takano and K. Nakagawa, "Sideband Suppression Characteristics of Optical SSB Generation Filter with Sampled FBG Based 4-taps Optical Hilbert Transformer," in Proceedings of the 15th Asia-Pacific Conference on Communications (APCC, 2009), pp. 622-625.

5. C. Sima, J. C. Gates, H. L. Rogers, P. L. Mennea, C. Holmes, M. N. Zervas, and P. G. R. Smith, "Phase controlled integrated interferometric single-sideband filter based on planar Bragg gratings implementing photonic Hilbert transform," Opt. Lett., 38, 727-729 (2013).

6. M. H. Asghari and J. Azaña, "All-optical Hilbert transformer based on a single phase-shifted fiber Bragg grating: Design and analysis," Opt. Lett., 34, 334-336 (2009).

7. M. Li and J. Yao, "Experimental Demonstration of a Wideband Photonic Temporal Hilbert Transformer Based on a Single Fiber Bragg Grating," IEEE Photon. Technol. Lett., 22, 1559 (2010).

8. L. Zhuang, M. R. Khan, W. Beeker, A. Leinse, R. Heideman, and C. Roeloffzen, "Novel microwave photonic fractional Hilbert transformer using a ring resonator-based optical all-pass filter," Opt. Express, 20, 26499 (2012).

9. R. Ashrafi, and J. Azaña, "Terahertz bandwidth alloptical Hilbert transformers based on long-period gratings," Optics Letters, 37(13), 2604 (2012).

10. C. Sima, J. C. Gates, M. N. Zervas, and P. G.R. Smith, "Review of photonic Hilbert transformers," Frontiers of Optoelectronics, 6,(1), 78-88. (2013).

11. C. Sima, J. C. Gates, P. L. Mennea, C. Holmes, M. N. Zervas, and P. G. R. Smith, "Ultra-wide detuning planar Bragg grating fabrication technique based on direct UV grating writing with electro-optic phase modulation", Opt. Express, 21(13), 15747-15754 (2013).

12. G. D. Emmerson, S. P. Watts, C. B. Gawith, V. Albanis, M. Ibsen, R. B. Williams, and P. G. R. Smith "Fabrication of directly UV-written channel waveguides with simultaneously defined integral Bragg gratings", Electron. Lett, 38, 1531-1532 (2002).

13. P. A. Williams, "Modulation phase-shift measurement of PMD using only four launched polarisation states: a new algorithm," Electron. Lett., 35, 1758(1999).

14. R. Feced, M. N. Zervas, and M. A. Muriel, "An efficient inverse scattering algorithm for the design of nonuniform fiber Bragg gratings," J. Quantum Electron., $35,1105-1115$ (1999).

15. R. Schmogrow, M. Winter, M. Meyer, D. Hillerkuss, S. Wolf, B. Baeuerle, A. Ludwig, B. Nebendahl, S. BenEzra, J. Meyer, M. Dreschmann, M. Huebner, J. Becker, C. Koos, W. Freude, and J. Leuthold, "Real-time Nyquist pulse generation beyond $100 \mathrm{Gbit} / \mathrm{s}$ and its relation to OFDM," Optics Express, 20(1), 317-337 (2012).

16. Masataka Nakazawa, Toshihiko Hirooka, Peng Ruan, and Pengyu Guan, "Ultrahigh-speed "orthogonal" TDM transmission with an optical Nyquist pulse train," Optics Express, 20(2), 1129-1140 (2012). 